\title{
Quantum spin pumping at a fractionally quantized magnetization state for a system with competing exchange interactions
}

\author{
Sujit Sarkar ${ }^{1,2}$ and C. D. Hu ${ }^{2}$ \\ ${ }^{1}$ PoornaPrajna Institute of Scientific Research, 4 Sadashivanagar, Bangalore 5600-80, India \\ ${ }^{2}$ Department of Physics, The National Taiwan University, Taipei 10617, Taiwan, Republic of China \\ (Received 15 October 2007; revised manuscript received 2 January 2008; published 12 February 2008)
}

\begin{abstract}
We study the quantum spin pumping of an antiferromagnetic spin-1/2 chain with competing exchange interactions. We show that spatially periodic potential modulated in space and time acts as a quantum spin pump. In our model system, an applied electric field causes a spin gap to its critical ground state by introducing bond-alternation exchange interactions. We study quantum spin pumping at different quantized magnetization states and also explain physically the presence and absence of quantum spin pumping at different fractionally quantized magnetization states.
\end{abstract}

DOI: 10.1103/PhysRevB.77.064413

PACS number(s): 75.10.Jm

\section{INTRODUCTION}

An adiabatic quantum pump is a device that generates a dc by a cyclic variation of some system parameters, the variation being slow enough so that the system remains close to the ground state throughout the pumping cycle. The pumping physics gets more attraction after the pioneering work of Thouless and Niu. ${ }^{1,2}$ Quantum adiabatic pumping physics is not only related to the spin system but also related to the other systems such as open quantum dots, ${ }^{3-5}$ superconducting quantum wires, ${ }^{6,7}$ the Luttinger quantum wire, ${ }^{8}$ and also to the interacting quantum wire. ${ }^{9}$

The motivation of our study is as follows: we have understood from the previous paragraph that adiabatic quantum pumping may arise in different systems due to the presence of different pumping sources. Here, we would like to study the adiabatic quantum pumping of a system that has not covered in any one of the previous studies. We consider an antiferromagnetic spin-1/2 chain with competing exchange interactions to study the adiabatic quantum spin pumping. We consider both nearest-neighbor (NN) and next-nearestneighbor (NNN) exchange interactions in the spin chain and only consider the presence of electric field that induces time dependent dimerization in both the exchange interactions. We also study the spin pumping at different states of magnetization. Our approach is completely analytical. We use the Abelian bosonization and one-loop renormalization group (RG) calculation to explore spin-pumping physics of this model Hamiltonian system. Shindou ${ }^{10}$ has studied only the Heisenberg $X X Z$ spin chain with $\mathrm{NN}$ dimerization. There are no competing exchange interactions and also the effect of different states of magnetization on adiabatic spin-pumping physics is absent. Shindou ${ }^{10}$ has considered two perturbations which open a gap in the excitation spectrum. One of them is the bond-alternation exchange interaction which leads to the dimerized state and the other one is the staggered magnetic field which locks the spin into a Néel ordered state. In his model, applied cyclic electric and magnetic fields control staggered component of exchange interaction and staggered magnetic field, respectively. In our case, there is only one perturbation which opens gap in the excitation spectrum. A part of our model has some experimental relevance. ${ }^{11,12}$
Suppose we have a spin-1/2 chain (such as $\mathrm{Cu}$-benzoate and the charge order phase of $\mathrm{Yb}_{4} \mathrm{As}_{3}$ ) with unit cell containing two crystallographic inequivalent sites, where both the translation symmetry $\left(T S_{j} \rightarrow S_{j+1}\right)$ and the bond centered inversion symmetry $\left(I_{b o n d} S_{i-j} \leftrightarrow S_{i+j+1}\right)$ are crystallographically broken. However, the NN exchange interaction $J$ in this spin chain does not have any alternating component because the system has site-centered inversion symmetry which exchanges the NN bonds. If we apply electric field in a particular direction of the system, ${ }^{13}$ then we may break the sitecentered inversion symmetry and it yields the bondalternation component in the $\mathrm{NN}$ exchange interaction. The additional interactions such as, NNN exchange interaction and it's alternating components in the Hamiltonians are completely theoretical. We consider these terms in the Hamiltonians to study the nontrivial and interesting effects of these terms over the basic interactions. In Sec. II, we present the model Hamiltonians and general derivations. Different sections are for the different states of magnetization. Section III is devoted for conclusions.

\section{MODEL HAMILTONIANS AND CONTINUUM FIELD THEORETICAL STUDY}

In our model Hamiltonians, we consider the presence of time dependent bond-alternation (dimerization) in both $\mathrm{NN}$ and NNN exchange interactions. We assume that the time dependence of dimerization is restored by the applied alternating electric field. In this section, we do all calculations, different sections are for the special limit of this general derivations. Our model Hamiltonians are as follows:

$$
\begin{aligned}
H_{A}= & J_{1} \sum_{n}\left[S_{n}(x) S_{n+1}(x)+S_{n}(y) S_{n+1}(y)+\Delta S_{n}(z) S_{n+1}(z)\right] \\
& +J_{2} \sum_{n}\left[1-\delta(t)(-1)^{n}\right] \vec{S}_{n} \cdot \vec{S}_{n+2}-g \mu_{B} H \sum_{n} S_{n}(z),
\end{aligned}
$$




$$
\begin{aligned}
H_{B}= & J_{1} \sum_{n}\left[1-\delta(t)(-1)^{n}\right]\left[S_{n}(x) S_{n+1}(x)+S_{n}(y) S_{n+1}(y)\right. \\
& \left.+\Delta S_{n}(z) S_{n+1}(z)\right]+J_{2} \sum_{n} \vec{S}_{n} \cdot \vec{S}_{n+2}-g \mu_{B} H \sum_{n} S_{n}(z),
\end{aligned}
$$

where $n$ is the site index and $x, y$, and $z$ are components of spin. Here, $J_{1}$ and $J_{2}$ are the nearest-neighbor and nextnearest-neighbor exchange couplings between spins, $J_{1}, J_{2}$ $\geqslant 0, \Delta$ is the $z$ component anisotropy of $\mathrm{NN}$ exchange interaction, $\delta(t)$ is the dimerization strength, which appears as a time dependent parameter in our Hamiltonians, and $H$ is the externally applied static magnetic field in the $z$ direction. The staggered component of exchange interaction is arising due to the broken site-centered inversion symmetry under an electric field in a particular direction. ${ }^{10} \mathrm{~A}$ site-centered inversion operation with the sign of elecrtic field reversed that requires $\delta$ must be an odd function of electric field. ${ }^{10}$

One can express spin-chain systems to a spinless fermion systems through the application of the Jordan-Wigner transformation. In the Jordan-Wigner transformation, the relations between the spin and the electron creation and annihilation operators are $S_{n}^{z}=\psi_{n}^{\dagger} \psi_{n}-1 / 2, S_{n}^{-}=\psi_{n} \exp \left[i \pi \Sigma_{j=-\infty}^{n-1} n_{j}\right]$, and $S_{n}^{+}$ $=\psi_{n}^{\dagger} \exp \left[-i \pi \sum_{j=-\infty}^{n-1} n_{j}\right]$, where $n_{j}=\psi_{j}^{\dagger} \psi_{j}$ is the fermion number at site $j$.

$$
\begin{aligned}
H_{A 1}= & -\frac{J_{1}}{2} \sum_{n}\left(\psi_{n+1}^{\dagger} \psi_{n}+\psi_{n}^{\dagger} \psi_{n+1}\right)+J_{1} \Delta \sum_{n}\left(\psi_{n}^{\dagger} \psi_{n}-1 / 2\right) \\
& \times\left(\psi_{n+1}^{\dagger} \psi_{n+1}-1 / 2\right)-g \mu_{B} H \sum_{n}\left(\psi_{n}^{\dagger} \psi_{n}-1 / 2\right), \\
H_{A 2}= & J_{2} \sum_{n}\left(\psi_{n+2}^{\dagger} \psi_{n}+\text { H.c. }\right)\left(\psi_{n+1}^{\dagger} \psi_{n+1}-1 / 2\right)+J_{2} \sum_{n}\left(\psi_{n}^{\dagger} \psi_{n}\right. \\
& -1 / 2)\left(\psi_{n+2}^{\dagger} \psi_{n+2}-1 / 2\right), \\
H_{A 3}= & -J_{2} \delta(t) \sum_{n}(-1)^{n}\left(\psi_{n+2}^{\dagger} \psi_{n}+\text { H.c. }\right)\left(\psi_{n+1}^{\dagger} \psi_{n+1}-1 / 2\right) \\
& -J_{2} \delta(t) \sum_{n}(-1)^{n}\left(\psi_{n}^{\dagger} \psi_{n}-1 / 2\right)\left(\psi_{n+2}^{\dagger} \psi_{n+2}-1 / 2\right) .
\end{aligned}
$$

There is a difference between the first term of Eq. (3) with the first terms of Eqs. (4) and (5). This difference arises due to the presence of an extra factor $e^{-i \pi n_{j+1}}$ in the string of the Jordan-Wigner transformation for NNN exchange interactions.

Similarly, one can also recast the spin-chain systems with $J_{1}$ dimerization into the spinless fermions. The Hamiltonians are converted as follows: $H_{B 1}=H_{A 1}, H_{B 2}=H_{A 2}$, and

$$
\begin{aligned}
H_{B 3}= & \frac{J_{1}}{2} \delta(t) \sum_{n}(-1)^{n}\left(\psi_{n+1}^{\dagger} \psi_{n}+\psi_{n}^{\dagger} \psi_{n+1}\right)+J_{1} \Delta \delta(t) \\
& \times \sum_{n}(-1)^{n}\left(\psi_{n+1}^{\dagger} \psi_{n+1}-1 / 2\right)\left(\psi_{n}^{\dagger} \psi_{n}-1 / 2\right) .
\end{aligned}
$$

Here, our Hamiltonians are different from previously studied dimerization problem. Haldane and White and Affleck have studied intrinsic dimerization for frustrated spin- $1 / 2$ antifer- romagnetic chain. In these studies, there is no explicit dimerization. Totsuka ${ }^{16}$ and Tonegawa et al. ${ }^{17}$ have studied the $H_{B}$ Hamiltonian only. There is no spin-pumping physics in any one of the previous studies. ${ }^{14-17}$ There are few other studies ${ }^{18-20}$ based on model $H_{A}$, but there is no spin-pumping physics in any one of these studies. So the current work is more wide and advance. Our approach is completely analytical; i.e., we explain the basic understanding of spin-pumping physics of our model system. Before we proceed further for continuum field theoretical study of these model Hamiltonians, we would like to explain the basic aspects of quantum spin pump of our model Hamiltonians: An adiabatic sliding motion of one dimensional potential, in gapped Fermi surface (insulating state), pumps an integer numbers of fermions per cycle. In our case, the transport of the Jordan-Wigner fermions (spinless fermions) is nothing but the transport of spin from one end of the chain to the other end because the number operator of spinless fermions is related with the $z$ component of spin density. ${ }^{21}$ We shall see that nonzero $\delta(t)$ introduces the gap at around the Fermi point and the system is in the insulating state (Peierls insulator). In this phase, spinless fermions form the bonding orbital between the neighboring sites, which yields a valence band in the momentum space. It is well known that the physical behavior of the system is identical at these two Fermi points. From the seminal paper of Berry, ${ }^{22}$ one can analyze this double degeneracy point. It appears as source and sink vector fields defined in the generalized crystal momentum space. ${ }^{22}$ Here, $B_{n}(K)=\nabla_{K} \times A_{n}(K)$ and $A_{n}(K)=\frac{i}{2 \pi}\left\langle n(K)\left|\nabla_{K}\right| n(K)\right\rangle$, where $K$ $=[k, \delta(t)]$. Here, $B_{n}$ and $A_{n}$ are the fictitious magnetic field (flux) and vector potential of the $n$th Bloch band, respectively. The degenerate points behave as a magnetic monopole in the generalized momentum space, whose magnetic unit can be shown to be $1,{ }^{10}$ analytically

$$
\int_{S_{1}} d S B_{ \pm}= \pm 1
$$

Positive and negative signs of the above equation are, respectively, for the conduction and valence bands. Conduction and valence bands meet at the degeneracy points. $S_{1}$ represent an arbitrary closed surface which encloses the degeneracy point. In the adiabatic process, the parameter $\delta(t)$ is changed along a loop $(\Gamma)$ enclosing the origin (minima of the system). It is well known in the literature of adiabatic quantum pumping physics that two independent parameters are needed to achieve the adiabatic quantum pumping in a system. ${ }^{3}$ Here, one may consider these two parameters as the real and imaginary parts of the Fourier transform of dimerized potential. When the shape of the dimerized potential will change in time, then it amounts to change the phase and amplitude in time. The role of adiabatic parameters is not explicit in our study. We define the expression for spin current $(I)$ from the analysis of the Berry phase. Then, according to the original idea of quantum adiabatic particle transport, ${ }^{1,2,10,23}$ the total number of spinless fermions (I) which are transported from one side of this system to the other is equal to the total flux of the valence band, which 
penetrates the two dimensional closed sphere $\left(S_{2}\right)$ spanned by the $\Gamma$ and the Brillioun zone. ${ }^{10}$

$$
I=\int_{S_{2}} d S B_{+1}=1
$$

We have already understood that quantized spinless fermion transport is equivalent to the spin transport. ${ }^{21}$ We will interpret this equation more physically at the end [Eq. (18)] of the next section. This quantization is topologically protected against the other perturbation as long as the gap along the loop remains finite. ${ }^{10,23}$

In the following paragraph, we do the continuum field theoretical studies of spin pumping for different magnetization states and explain the stabilization of quantized spin pumping against the $z$ component of exchange interactions and also from the intrinsic dimerization [when $J_{2}>0.241 J_{1}$ (Ref. 24)]. We recast the spinless fermion operators in terms of field operators by this relation,

$$
\psi(x)=\left[e^{i k_{F} x} \psi_{R}(x)+e^{-i k_{F} x} \psi_{L}(x)\right],
$$

where $\psi_{R}(x)$ and $\psi_{L}(x)$ describe the second-quantized fields of right- and left-moving fermions, respectively. In the absence of magnetic field $(H=0), k_{F}= \pm \pi / 2$; however, we are interested to study the systems in the presence of static magnetic field. Therefore, we keep the Fermi momentum as arbitrary $k_{F}$. One can simply absorb the finite magnetization in a shift of field $\phi$ by $\phi=\widetilde{\phi}-\pi m x$, where $m=\left\langle S_{z}\right\rangle$. In presence of magnetic field, the Fermi momentum and magnetization $(m)$ are related by this relation, $k_{F}=\frac{\pi}{2}(1-2 m) .{ }^{25}$ We want to express the fermionic fields in terms of bosonic field by this relation,

$$
\psi_{r}(x)=\frac{U_{r}}{\sqrt{2 \pi \alpha}} e^{-i[r \phi(x)-\theta(x)]} .
$$

Here, $r$ is denoting the chirality of the fermionic fields, right (1) or left movers $(-1)$. The operators $U_{r}$ are operators that commute with the bosonic field. $U_{r}$ of different species commute and $U_{r}$ of the same species anticommute. $\phi$ field corresponds to the quantum fluctuations (bosonic) of spin and $\theta$ is the dual field of $\phi$. They are related by these relations, $\phi_{R}=\theta-\phi$ and $\phi_{L}=\theta+\phi$.

Using the standard machinery of continuum field theory, ${ }^{25}$ we finally get the bosonized Hamiltonians as

$$
\begin{aligned}
H_{0}= & v_{0} \int_{o}^{L} \frac{d x}{2 \pi}\left\{\pi^{2}: \Pi^{2}:+:\left[\partial_{x} \phi(x)\right]^{2}:+\frac{g_{1}}{\pi^{2}} \int d x:\left[\partial_{x} \phi_{L}(x)\right]^{2}:\right. \\
& +:\left[\partial_{x} \phi_{R}(x)\right]^{2}:+\frac{g_{2}}{\pi^{2}} \int d x\left[\partial_{x} \phi_{L}(x)\right]\left[\partial_{x} \phi_{R}(x)\right] .
\end{aligned}
$$

Here, $H_{0}$ is the gapless Tomonoga-Luttinger liquid part of the Hamiltonian with $v_{0}=\sin k_{F}$. The analytical expressions for $g_{1}$ and $g_{2}$ (related with the forward scattering of fermionic field) are as follows: $g_{1}=2\left(\Delta-2 J_{2}\right) \sin ^{2} k_{F}$ $+2 J_{2} \sin 2 k_{F}\left(\pi+\sin 2 k_{F}\right) \quad$ and $\quad g_{2}=4\left(\Delta-2 J_{2}\right) \sin ^{2} k_{F}$ $+4 J_{2} \sin ^{2} 2 k_{F}$.

Analytical expressions for different exchange interactions of Hamiltonian $H_{A}$ are as follows:

$$
H_{J 2 C 1}=\frac{J_{2}}{2 \pi^{2} \alpha^{2}} \int d x: \cos \left[4 \sqrt{K} \phi(x)-\left(G-4 k_{F}\right) x-4 k_{F} a\right]:
$$

$$
H_{J 2 C 2}=\frac{J_{1} \Delta}{2 \pi^{2} \alpha^{2}} \int d x: \cos \left[4 \sqrt{K} \phi(x)+\left(G-4 k_{F}\right) x-2 k_{F} a\right]:
$$

$$
H_{J 2 C 3}=\frac{J_{2} \delta(t)}{2 \pi^{2} \alpha^{2}} \int d x: \cos \left[\left(\pi-4 k_{F}\right) x+4 \sqrt{K} \phi(x)-4 k_{F} a\right]:
$$

where $G$ is the reciprocal lattice vector. Equations (12) and (13) present the UMKLAPP scattering term from the NN and NNN antiferromagnetic exchange interactions; Eq. (14) appears due to the presence of dimerized interaction. Similarly, one can also find the expressions for $H_{B}$ Hamiltonian. Analytical expressions for $K$ is as follows:

$$
K=\left[\frac{1-(8 / \pi) J_{2} \sin ^{2} k_{F}+4 J_{2} \cos k_{F}}{1+(4 / \pi) \Delta \sin k_{F}+4 J_{2} \cos k_{F}\left(1+2 / \pi \sin 2 k_{F}\right)}\right]^{1 / 2} .
$$

Here, $v_{0}$ and $K$ are the two Luttinger liquid parameters. During this derivation, we have used the following relations: $\rho_{R / L}=\frac{-1}{\pi} \partial_{x} \phi_{R / L}(x)$ and $[\phi(x), \Pi(x)]=i \delta\left(x-x^{\prime}\right)$, where $\Pi(x)$ $=\frac{1}{\pi} \nabla \theta(x)$ is the canonically conjugate momentum. We have also used the following equation: $S^{z}(x)=a\left[\rho(x)+(-1)^{j} M(x)\right]$. The bosonized expressions for $\rho$ and $M$ are given by $\rho(x)$ $=-\frac{1}{\sqrt{\pi}} \partial_{x} \phi(x)$ and $M(x)=\frac{1}{\pi a} \cos [2 \phi(x)]$. Similarly, one can calculate the analytical expressions for $J_{1}$ dimerization. Here, we have expressed our all expressions in terms of bare phase field $(\phi)$ by using the conventional practice of continuum field theory. ${ }^{25}$ During these derivations, we assume that $J_{1}$ $\gg J_{2}, \delta . J_{2}$ is in the unit of $J_{1}$. Here, we neglect the higher order of $a$ than $a^{2}$.

\section{A. Calculations and results for $M=0$ magnetization states}

At first, we discuss $m=0$ magnetization state; it corresponds to $k_{F}= \pm \pi / 2$. Here, we study both the effect of $X X Z$ anisotropy $(\Delta)$ and the spin-Peierls dimerization $[\delta(t)]$. The effective Hamiltonian for $J_{2}$ dimerization becomes

$$
H_{A}=H_{0}+\left(\frac{J_{2}-J_{1} \Delta}{2 \pi^{2} \alpha^{2}}\right) \int d x: \cos [4 \sqrt{K} \phi(x)]: .
$$

In this effective Hamiltonian [Eq. (16)], there is no contribution from dimerized interaction $\left[k_{F}=\pi / 2\right.$ limit of Eq. (14)] due to the oscillatory nature of the integrand (it leads to the vanishing contribution). However, the contribution of dimerized potential is present in the NN exchange interaction. Similarly, the effective Hamiltonian for $J_{1}$ dimerization becomes 


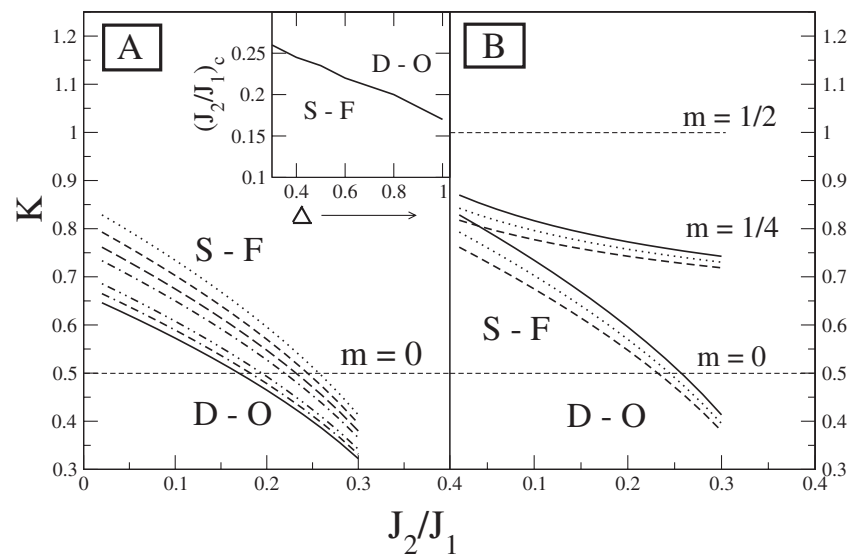

FIG. 1. Luttinger liquid parameter $(K)$ versus $\frac{J_{2}}{J_{1}}$ for different magnetization state. A constant dashed line at $K=0.5$ is for eye guideline, which separating the spin fluid and dimer order instability state. D-O: dimer order instability state, S-F: spin-fluid Luttinger liquid phase. (A) Here, we only focus at $m=0$ magnetization state. Uppermost (dotted) curve is for $\Delta=0.3$ and the lowermost (solid) curve is for $\Delta=1$. The intermediate curves are for $\Delta$ $=0.4,0.5,0.6,0.8,0.9$, respectively, from upper to lower one. The inset shows the separation between the spin fluid and dimer order instability state by a critical line. Here, we present the shift of the $\left(\frac{J_{2}}{J_{1}}\right)_{c}$ with $\Delta$. (B) Here, we present the curves for different magnetization states. $m=1 / 2$ is independent of $\Delta$ and $\frac{J_{2}}{J_{1}}$ in contrast with $m=1 / 4,0$. The curves for $m=1 / 4,0$ plateaus are for $\Delta$ $=0.4,0.5,0.6$, respectively, from upper to lower one.

$$
\begin{aligned}
H_{B}= & H_{0}+\left(\frac{J_{2}-J_{1} \Delta}{2 \pi^{2} \alpha^{2}}\right) \int d x: \cos [4 \sqrt{K} \phi(x)]: \\
& +\frac{J_{1} \delta(t)}{2 \pi^{2} \alpha^{2}} \int d x: \cos [2 \sqrt{K} \phi(x)]:
\end{aligned}
$$

This dimerization contribution for $\mathrm{NN}$ exchange interaction has originated from the $X Y$ interaction. This dimerization is the spontaneous dimerization; i.e., infinitesimal amount of $\delta(t)$ is sufficient to produce a gap around the Fermi points. The other two contributions of $J 1$ dimerization are from the $X X Z$ anisotropy of NN exchange interaction and the $z$ component of NNN interaction. Figure 1 shows the variation of $K$ with $\frac{J_{2}}{J_{1}}$ for different values of $\Delta$. We observe from the figure that $K$ is the function of $\Delta$ for fixed $\frac{J_{2}}{J_{1}}$. (The inset of Fig. 1 shows the appearence of intrinsic dimerization as a function of $\frac{J_{2}}{J_{1}}$ and $\Delta$. There are a few studies ${ }^{26,27}$ on the phase seperation between the spin-fluid and dimer order phases of frustrated spin chain, but our Hamiltonians are different from them.) The second term of Eq. (17) is irrelevant when $K$ is greater than $1 / 2$. So in this parameter space, only the time dependent dimerizing field [third term of Eq. $(17)]$ is relevant and lock the phase operator at $\phi=0+\frac{n \pi}{\sqrt{K}}$. Now, the locking potential slides adiabatically (here, the cyclic electric field that produces the dimerization). Speed of the sliding potential is low enough such that system stays in same valley; i.e., there is no scope to jump onto the other valley. The system will acquire $2 \pi$ phase during one complete cycle of external electric field around the loop encircling the minima of critical ground state, produced by the dimerizing field. This is the basic mechanism of spin pumping of our system. This expection is easily verified when we notice the physical meaning of the phase operator $[\phi(x)]$. Since the spatial derivative of the phase operator corresponds to the $z$ component of spin density, this phase operator is nothing but the difference of the spatial polarization of the $z$ component of spin, i.e., $P_{s^{z}}=-\frac{1}{N} \sum_{j=1}^{N} j S_{j}^{z}$. Shindou has shown explicitly the equivalence between these two considerations. ${ }^{10}$ During the adiabatic process, $\left\langle\phi_{t}\right\rangle$ changes monotonically and acquires $-2 \pi$ phase. In this process, $P_{s}^{z}$ increases by 1 / cycle. We define it analytically as

$$
\delta P_{s}^{z}=\int_{\Gamma} d P_{s}^{z}=-\frac{1}{2 \pi} \int d x \partial_{x}\langle\phi(x)\rangle=1 .
$$

This physics always holds as far as the system is locked by the sliding potential and $\Delta<1 .^{10}$ This equation [Eq. (18)] for spin transport is physically consistent with Eq. (7) (based on the Berry phase analysis) of spin current. The quantized spin transport of this scenario can be generalized up to the value of $\Delta$ for which $K$ is greater than $1 / 2$. In this limit, $z$ component of exchange interaction and also the intrinsic dimerization has no effect on the spin-pumping physics of applied electric field induced dimerized interaction of $H_{B}$ Hamiltonian.

\section{B. Calculations and results for $m=\frac{1}{4}$ magnetization states}

Here, we discuss the physics of quantum spin pumping of a finite magnetization state. We are considering the magnetization state at $m=\frac{1}{4}$; it corresponds to $k_{F}= \pm \frac{\pi}{4}$. The effective Hamiltonian for $J_{2}$ dimerization becomes

$$
\begin{aligned}
H_{A}= & H_{0}-\left(\frac{J_{1} \delta(t)}{2 \pi^{2} \alpha^{2}}\right) \int d x: \cos [4 \sqrt{K} \phi(x)]: \\
& +\frac{A}{2 \pi^{2} \alpha^{2}} \int d x: \cos [4 \sqrt{K} \phi(x)+\beta]:
\end{aligned}
$$

where $A=\sqrt{J_{1}^{2} \Delta^{2}+J_{2}^{2}}$ and $\beta=\tan ^{-1} \frac{J_{1} \Delta}{J_{2}}$. Apparently, it appears from the general derivation of Sec. II, which the second and third terms of Eq. (19) will be absent due to the oscillatory nature of the integrand, but this is not the case when one considers the dimerized lattice. In dimerized lattice, reciprocal lattice vector $G$ will change from $2 \pi$ to $\pi$ due to the change of the size of the unit cell. It becomes more clear if one writes these terms as $\int d x: \cos \left[\left(G-4 k_{F}\right) x+4 \sqrt{K} \phi(x)\right]$ :

Similarly, one can write the effective Hamiltonian for $J_{1}$ dimerization,

$$
\begin{aligned}
H_{B}= & H_{0}+\left(\frac{J_{1} \Delta \delta(t)}{2 \pi^{2} \alpha^{2}}\right) \int d x: \sin [4 \sqrt{K} \phi(x)]: \\
& -\frac{A}{2 \pi^{2} \alpha^{2}} \int d x: \cos [4 \sqrt{K} \phi(x)+\beta]:
\end{aligned}
$$


The analytical structures of Eqs. (19) and (20) are the same; i.e., the coefficient of the field $\phi(x)$ is the same for all sineGordon coupling terms. The renormalization group equations for these types of interactions are $\mathrm{a}^{25,28}$

$$
\begin{aligned}
& \frac{d K}{d \ln L}=-4 \pi^{2} K^{2} \delta(t)^{2}, \\
& \frac{d \delta(t)}{d \ln L}=(2-4 K) \delta(t) .
\end{aligned}
$$

It appears from these RG equations that to get a relevant perturbation, $K$ should be less than $1 / 2$. It reveals from Fig. 1(b) that $K$ is exceeding the relevant value in our region of interest to mature criteria for spin pumping. So the dimerization strength should exceed some critical value $\left(\delta_{c}\right)$ to initiate the spin-pumping phase. These two equations are the Kosterlitz-Thousless equation for the system in this limit. At the critical point, system undergoes the Kosterlitz-Thouless transition. ${ }^{25,28}$ Since the system flows to the strong coupling (dimer order) as the dimerization strength exceeds some critical value $\left(\delta_{c}\right)$ initially, we have to guess the physics of this phase. We analyze the system in the limit $\delta \rightarrow \pm \infty$ and $K \rightarrow 0$. In this limit, all sine-Gordon couplings are relevant but the value of $\phi$ is pinned at the minima of $\cos (4 \sqrt{K} \phi)$ for NN dimerization and of $\sin (4 \sqrt{K} \phi)$ for NNN dimerization because the dimerization strength is larger than the other couplings of the system. Hence, it produces a deeper minima for the system. This parameter dependent transition, from massless phase to massive phase, at $T=0$ is the quantum phase transition. This quantum phase transition occurs at the every magnetization state. So we conclude that the appearance of quantum spin pumping is not spontaneous like $m$ $=0$, rather dependent on the strength of the parameter.

\section{Calculations and results for $m=\frac{1}{2}$ and others fractionally quantized magnetization states}

Now, we discuss the saturation magnetization at $m=\frac{1}{2}$ $\left(k_{F}=0\right)$. Here, $K_{F}=0$ implies that the band is empty and the dispersion is not linear, so the validity of the continuum field theory is questionable. Values of the two Luttinger liquid parameters, $v_{0}$ and $K$, are 0 and 1 , respectively. It also implies that none of the sine-Gordon coupling terms becomes relevant in this parameter space. So there is no spin pumping for these fractionally quantized magnetization states.

Here, we present the explanation for the absence of other fractionally quantized magnetization state (such as $1 / 3,1 / 5$, 1/7, etc): A careful examination of Eqs. (12)-(14) reveals that to get a nonoscillatory contribution from Hamiltonian, one has to be satisfied $4 k_{F}=G$ condition, but this condition is not fulfilled for these fractionally quantized magnetization state. There are no sine-Gordon coupling terms. Hence, there is no spin-pumping physics for these fractionally quantized states of magnetization.

\section{CONCLUSIONS}

We have presented the physics of quantum spin pump for different magnetization states of an antiferomagnetic spin$1 / 2$ chain with competing exchange interactions along with bond-alternation interactions. Our study is completely analytical. We have observed that for some magnetization state, spin pumping is spontaneous, and for some other, it is not and also explained the physical reasons for the presence and absence of spin pumping for those states.

\section{ACKNOWLEDGMENTS}

The author (S.S.) would like to acknowledge The Center for Condensed Matter Theory of IISc for providing the working space and The National Center for Theoretical Science (Taipei) where the initial phase of this work has started. Finally, the author thanks B. Mukhopadhyay for reading the paper very critically.
${ }^{1}$ D. J. Thouless, Phys. Rev. B 27, 6083 (1983).

${ }^{2}$ Q. Niu and D. J. Thouless, J. Phys. A 17, 2453 (1984).

${ }^{3}$ P. W. Brouwer, Phys. Rev. B 58, R10135 (1998).

${ }^{4}$ T. A. Shutenko, I. L. Aleiner, and B. L. Altshuler, Phys. Rev. B 61, 10366 (2000).

${ }^{5}$ Y. Levinson, O. Entin-Wohlman, and P. Wolfe, Physica A 302, 335 (2001); O. Entin-Wohlman and A. Aharony, Phys. Rev. B 66, 035329 (2002).

${ }^{6}$ M. Blaauboer, Phys. Rev. B 65, 235318 (2002).

${ }^{7}$ J. Wang and B. Wang, Phys. Rev. B 65, 153311 (2002); B. Wang and J. Wang, ibid. 66, 201305(R) (2002).

${ }^{8}$ P. Sharma and C. Chamon, Phys. Rev. Lett. 87, 096401 (2001); A. Crépieux, R. Guyon, P. Devillard, and T. Martin, Phys. Rev. B 67, 205408 (2003).

${ }^{9}$ R. Citro, N. Anderi, and Q. Niu, Phys. Rev. B 68, 165312 (2003).

${ }^{10}$ R. Shindou, J. Phys. Soc. Jpn. 74, 1214 (2005).

${ }^{11}$ M. Kohgi, K. Iwasa, J. M. Mignot, B. Fak, P. Gegenwart, M. Lang, A. Ochiai, H. Aoki, and T. Suzuki, Phys. Rev. Lett. 86,
2439 (2001).

${ }^{12}$ P. Fulde, B. Schmidt, and P. Thalmeier, Europhys. Lett. 31, 323 (1995).

${ }^{13}$ Here, the system is invariant under the $\pi$-rotational symmetry which exchange the NN bonds. The direction of electric field and $\pi$-rotational axis are different.

${ }^{14}$ F. D. M. Haldane, Phys. Rev. B 25, 4925 (1982).

${ }^{15}$ S. R. White and I. Affleck, Phys. Rev. B 54, 9862 (1996).

${ }^{16}$ K. Totsuka, Phys. Rev. B 57, 3454 (1998).

${ }^{17}$ T. Tonegawa et al., Physica B 246-247, 368 (1998).

${ }^{18}$ S. Chen, H. Buttner, and J. Voit, Phys. Rev. Lett. 87, 087205 (2001).

${ }^{19}$ L. Capriotti, F. Becca, S. Sorella, and A. Parola, Phys. Rev. Lett. 89, 149701 (2002).

${ }^{20}$ S. Sarkar and D. Sen, Phys. Rev. B 65, 172408 (2002).

${ }^{21} S_{n}^{z}=\frac{1}{2 \pi} \partial_{x} \phi\left(x_{n}\right)-\frac{(-1)^{n}}{\pi \alpha} \cos \left[\phi\left(x_{n}\right)\right]$. Here, $\phi$ field corresponds to the quantum fluctuations (boson) of spin. 
${ }^{22}$ M. V. Berry, Proc. R. Soc. London, Ser. A 392, 45 (1984).

${ }^{23}$ J. E. Avron, A. Raveh, and B. Zur, Rev. Mod. Phys. 60, 873 (1988); J. E. Avron, J. Berger, and Y. Last, Phys. Rev. Lett. 78, 511 (1997).

${ }^{24}$ R. Chitra, S. Pati, H. R. Krishnamurthy, D. Sen, and S. Ramasesha, Phys. Rev. B 52, 6581 (1995).
${ }^{25}$ T. Giamarchi, Quantum Physics in One Dimension (Clarendon Press, Oxford, 2004).

${ }^{26}$ K. Nomura and K. Okamoto, J. Phys. Soc. Jpn. 62, 1123 (1993).

${ }^{27}$ R. D. Somma and A. A. Aligia, Phys. Rev. B 64, 024410 (2001).

${ }^{28}$ J. M. Kosterlitz and D. J. Thouless, J. Phys. C 6, 1181 (1973); V. L. Berezinski, Sov. Phys. JETP 32, 493 (1971). 